\section{A case of primary oropharyngeal and gastric syphilis mimicking oropharyngeal cancer}
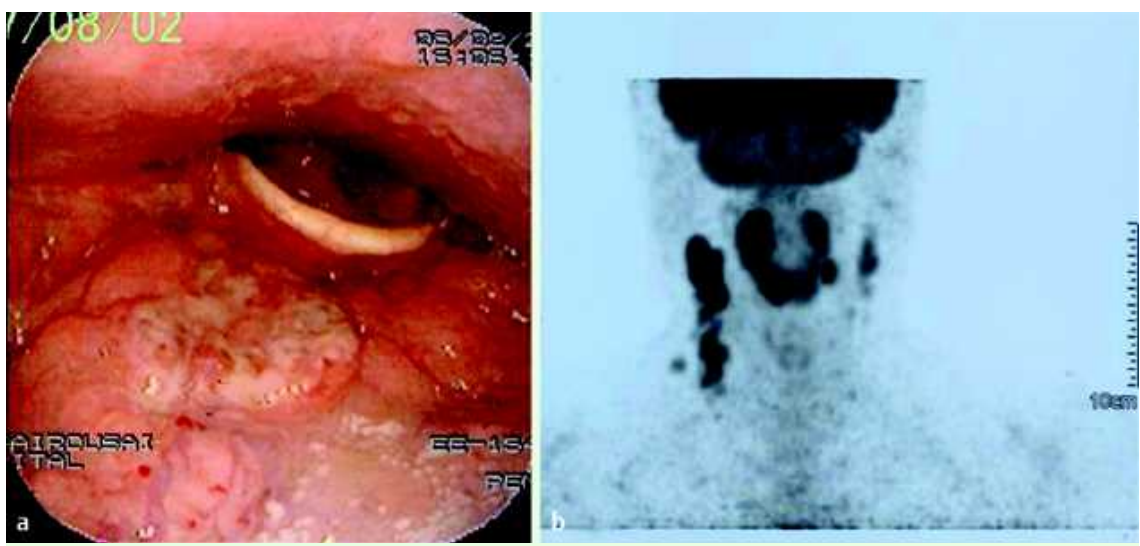

Fig. 1 Endoscopic nasopharyngeal findings (a) and FDG-PET image (b). A round elevated tumor was found in the anterior wall of the oropharynx (a). Increased FDG accumulation in the oropharyngeal tumor and lymph nodes of the cervical regions on both sides (b).
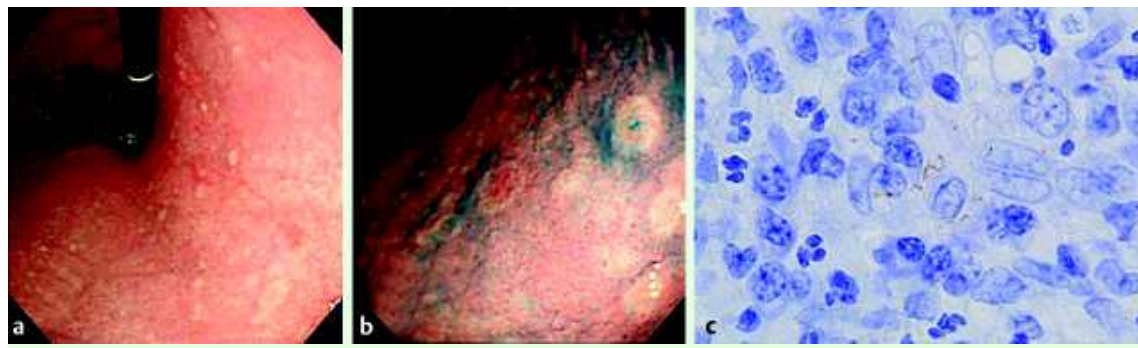

Fig. 2 Endoscopic findings in the upper body of the stomach $(\mathbf{a}, \mathbf{b})$ and histopathological finding (c). Multiple small round erosions with brownish halos, ranging from 5 to $10 \mathrm{~mm}$, were observed over the entire cardia and body, although there were no lesions in the antral and pyloric regions (b: indigo carmine staining). Histopathological

$\left[{ }^{18} \mathrm{~F}\right]$ Fluorodeoxyglucose uptake on positron emission tomography (FDG-PET) is one of the most valuable imaging methods by which to establish tumor extent and size, to assess nodal disease, and to detect distant metastasis in head and neck cancer. For this reason, FDG-PET imaging has been reported to be useful for preoperative or preradiotherapy staging $[1,2]$. However, high FDG uptake can be observed in inflammatory diseases [3-5]; thus, it is important to suspect inflammatory or infectious diseases even in cancer patients to avoid a false-positive interpretation. analysis using immunohistochemical technique (anti-Treponema pallidum polyclonal antibody, 1:500; AbD Serotec, Oxford, UK) revealed numerous spirochetes of Treponema pallidum in specimens from both the oropharynx and the stomach (c).

We report a case of oropharyngeal and gastric syphilis mimicking oropharyngeal cancer due to increased FDG uptake.

A 48-year-old man with an oropharyngeal tumor and cervical lymphadenopathy underwent a medical examination at our hospital. Although these lesions were strongly suggestive of oropharyngeal cancer judging from the nasopharyngeal findings ( $\bullet$ Fig. 1a), CT scan, and, especially, FDG-PET, which showed a high level of accumulation ( $\mathbf{F i g . 1 b}$ ), histological study of the oropharynx showed nonspecific inflammation without malignant cells.
Endoscopy was performed for the purpose of screening for synchronous upper gastrointestinal cancer. This showed multiple small round erosions with brownish halos, ranging from 5 to $10 \mathrm{~mm}$, over the entire cardia and body ( $\odot$ Fig. 2 a, b).

The endoscopic appearance was suspected to be due to gastric syphilis, and biopsy specimens from the oropharynx and the stomach proved the presence of spirochetes of Treponema pallidum using Warthin-Starry silver staining and immunohistochemical technique ( $\bullet$ Fig. 2 c). On the specific serological tests for Treponema pallidum, a rapid plasma regain (RPR) test was positive for a titer of $1: 64$ and Treponema pallidum hemagglutination (TPHA) revealed a titer of $1: 5120$. The patient was subsequently tested for the human immunodeficiency virus (HIV), with negative result.

The patient was diagnosed with oropharyngeal and gastric syphilis and treated with penicillin for a month.

Careful endoscopic examination could allow the correct diagnosis of oropharyngeal syphilis to be made despite the false-positive FDG-PET result.

Endoscopy_UCTN_Code_CCL_1AB_2AC_3AB Endoscopy_UCTN_Code_CCL_1AB_2AC_3AZ Endoscopy_UCTN_Code_CCL_1AB_2AD_3AB Endoscopy_UCTN_Code_CCL_1AB_2AD_3AC

S. Tamura ${ }^{1}$, Y. Takimoto ${ }^{2}$, Y. Hoshida ${ }^{3}$, K. Okada ${ }^{1}$, M. Yoshimura ${ }^{1}$, K. Uji ${ }^{1}$,

A. Yoshida ${ }^{1}$, H. Miki ${ }^{1}$, M. Itoh ${ }^{2}$ Department of Surgery, Kansai Rosai Hospital, Amagasaki, Japan

Department of Otorhinolaryngology, Kansai Rosai Hospital, Amagasaki, Japan Department of Pathology, Kansai Rosai Hospital, Amagasaki, Japan

\section{References}

1 Benchaou M, Lehmann W, Slosman DO et al. The role of FDG-PET in the preoperative assessment of $\mathrm{N}$-staging in head and neck cancer. Acta Otolaryngol 1996; 116: 332 335

2 Jeong HS, Beak CH, Son YI et al. Use of integrated 18F-FDG PET/CT to improve the accuracy of initial cervical nodal evaluation in patients with head and neck squamous cell carcinoma. Head Neck 2007; 29: 203 210

3 Fernandez P, Guyot M, Lazaro E et al. Systemic tuberculosis presenting as an epiglottic mass detected on F-18 FDG PET/CT. Clin Nucl Med 2007; 32: 719- 724 
4 Enomoto $K$, Hoshida $Y$, Hamada $K$ et al. F-18FDG PET imaging of cervical tuberculous lymphadenitis. Clin Nucl Med 2007; 32: $474-475$

5 Kawabe J, Okamura T, Shakudo M et al. Two cases of chronic tonsillitis studied by FDGPET. Ann Nucl Med 1999; 13: 277-279
Bibliography

DOI $10.1055 / \mathrm{s}-2008-1077679$

Endoscopy 2008; 40: E235-E236

(c) Georg Thieme Verlag KG Stuttgart · New York . ISSN 0013-726X
Corresponding author

\section{S. Tamura, MD}

Department of Surgery, Kansai Rosai Hospital 1-69 3-Chome, Inabaso

Amagasaki

Hyogo 660-8511

Japan

Fax: +81-6-6419-1870

stamura@kanrou.net 